Supporting Information

\title{
Reversible Underwater Adhesion for Soft Robotic Feet by Leveraging Electrochemically Tunable Liquid Metal Interfaces
}

Hongda Lu $u^{\dagger}$, Guolin Yun ${ }^{\dagger}$, Tim Cole ${ }^{\star}$, Yiming Ouyang ${ }^{\perp}$, Hongtai Ren ${ }^{\perp}$, Jian Shu ${ }^{\perp}$, Yuxin

Zhang ${ }^{\ddagger}$, Shiwu Zhang* ${ }^{*}$, Michael D. Dickey*\$, Weihua Li*广, Shi-Yang Tang*t

$\dagger$ School of Mechanical, Materials, Mechatronic and Biomedical Engineering, University of

Wollongong, Wollongong, NSW 2522, Australia.

\$Department of Electronic, Electrical and Systems Engineering, University of Birmingham, Edgbaston, Birmingham, B15 2TT, UK.

$\perp$ CAS Key Laboratory of Mechanical Behavior and Design of materials, Department of

Precision Machinery and Precision Instrumentation, University of Science and Technology of China, Hefei 230026, China

$\S$ Department of Chemical and Biomolecular Engineering, North Carolina State University, Raleigh, NC 27695, USA.

Shi-Yang Tang - Email: $\underline{\text { S.Tang @bham.ac.uk }}$

Weihua Li - Email: weihuali@uow.edu.au

Michael D. Dickey - Email: mddickey@ncsu.edu

Shiwu Zhang - Email: swzhang@ ustc.edu.cn 


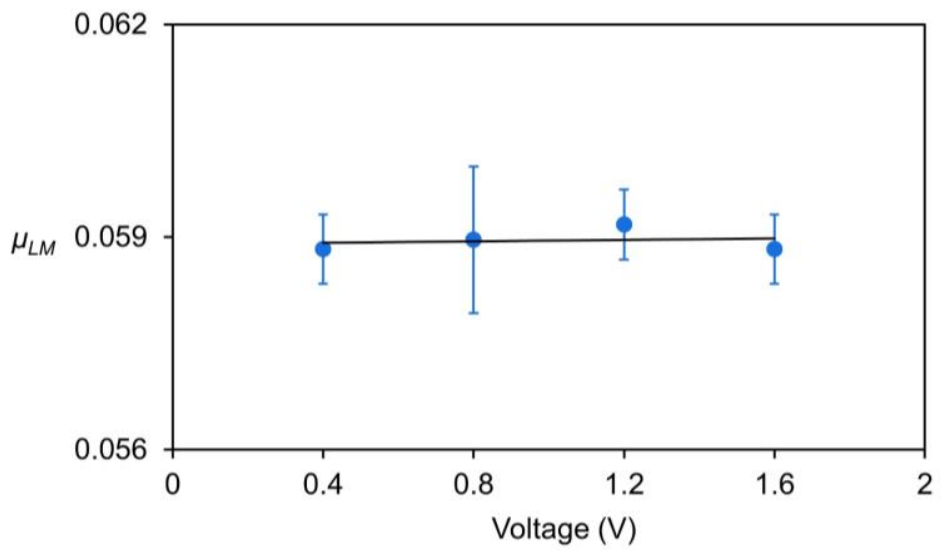

Figure S1. Coefficients of friction between the LM droplet and a glass surface. No obvious discrimination of the friction coefficient is observed when the voltage applied to the LM droplet varies from 0.4 to $1.6 \mathrm{~V}$. 

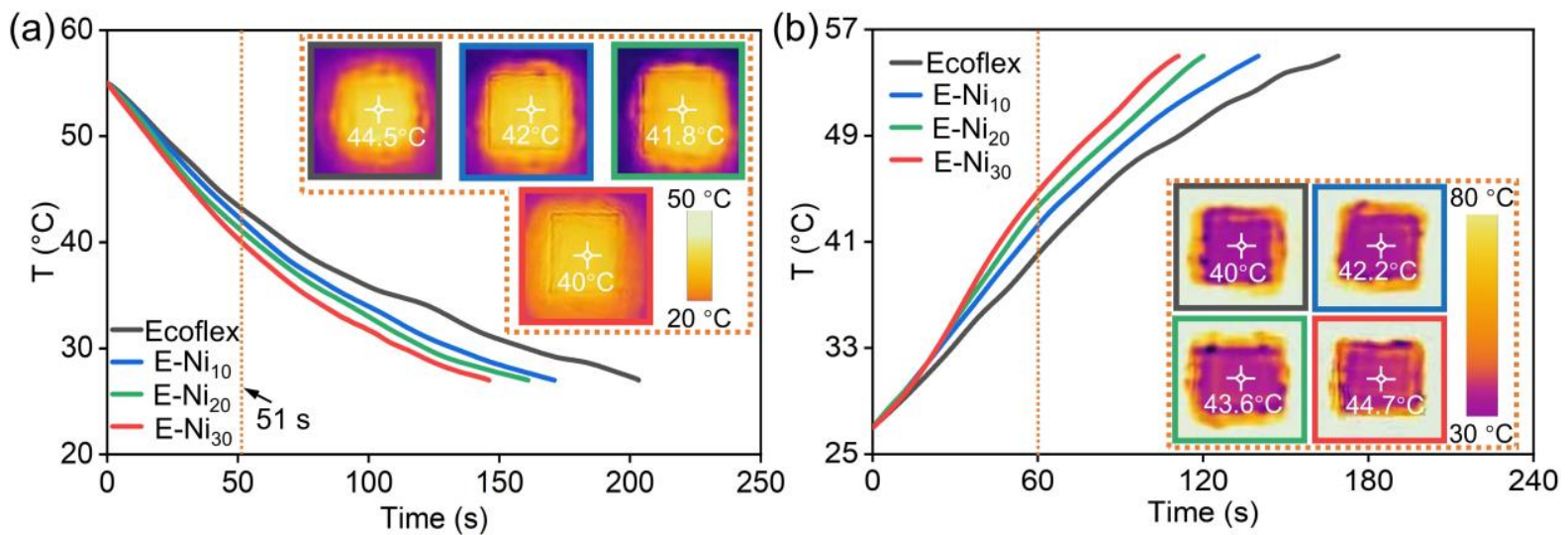

Figure S2. Characterization of the heat conductivities of Ecoflex composites with Ni particles embedded. a) Temperature $v s$ time plots for the composites containing different Ni ratios $(0 \%$ to $30 \%$ ). The inset shows thermal images of the four composite samples (length $\times$ width $\times$ height: $10 \times 10 \times 3 \mathrm{~mm}$ ) after cooling for $51 \mathrm{~s}$ from an initial temperature of $55^{\circ} \mathrm{C}$. The color of the border corresponds to the plot for different particle contents. b) Temperature $v s$ time plots for the four composite samples heated on a hot plate $\left(80^{\circ} \mathrm{C}\right)$ from 27 to $55^{\circ} \mathrm{C}$. The inset shows thermal images of the four composite samples after heating for $60 \mathrm{~s}$. The color of the border corresponds to the plot for different particle contents.

We fabricated Ecoflex composite samples (length $\times$ width $\times$ height: $10 \times 10 \times 3 \mathrm{~mm}$ ) with $10 \%, 20 \%, 30 \%$ weight ratios of $\mathrm{Ni}$ particles (denoted as $\mathrm{E}-\mathrm{Ni} \mathrm{i}_{10}, \mathrm{E}-\mathrm{Ni}_{20}, \mathrm{E}-\mathrm{Ni} \mathrm{i}_{30}$, respectively), which will not render electrically conductive. Figure S2a shows the cooling experiments conducted using the samples by setting the initial temperature at $55^{\circ} \mathrm{C}$. A high particle content leads to a high thermal conductivity, thereby giving a shorter cooling time. Similarly, the heating experiments given in Fig. S2b shows the same trend, where a composite with a high particle content leads to a faster heating time. However, the increase in thermal conductivity is not linearly proportional to the particle content, and the enhancement in thermal conductivity becomes less obvious when the weight ratio is above $20 \%$. 


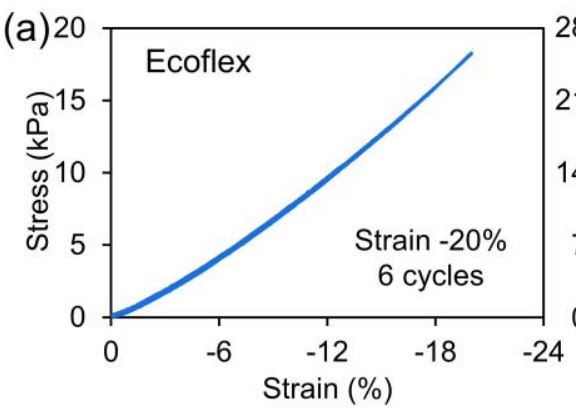

(b) 40

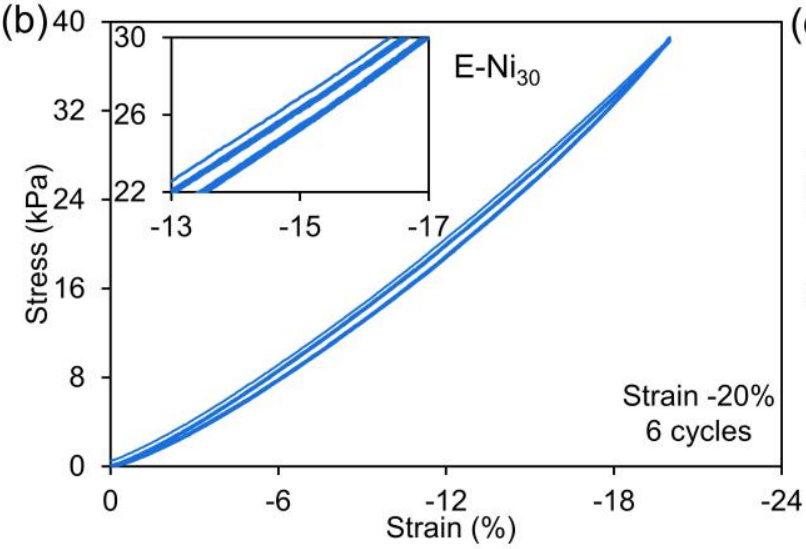

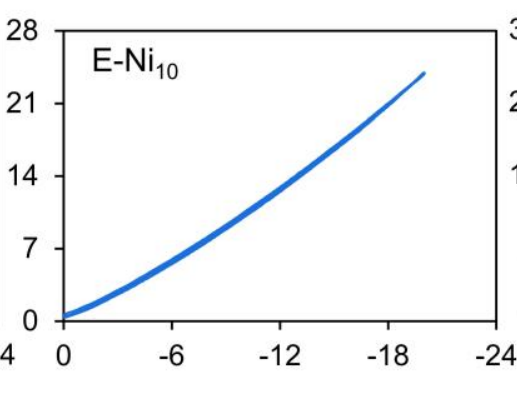

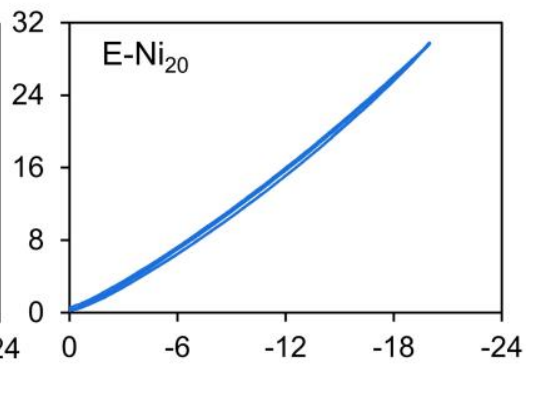

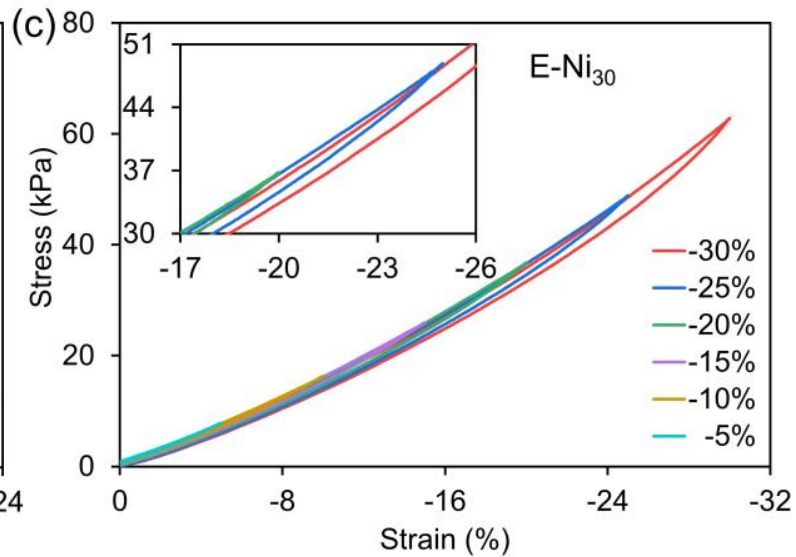
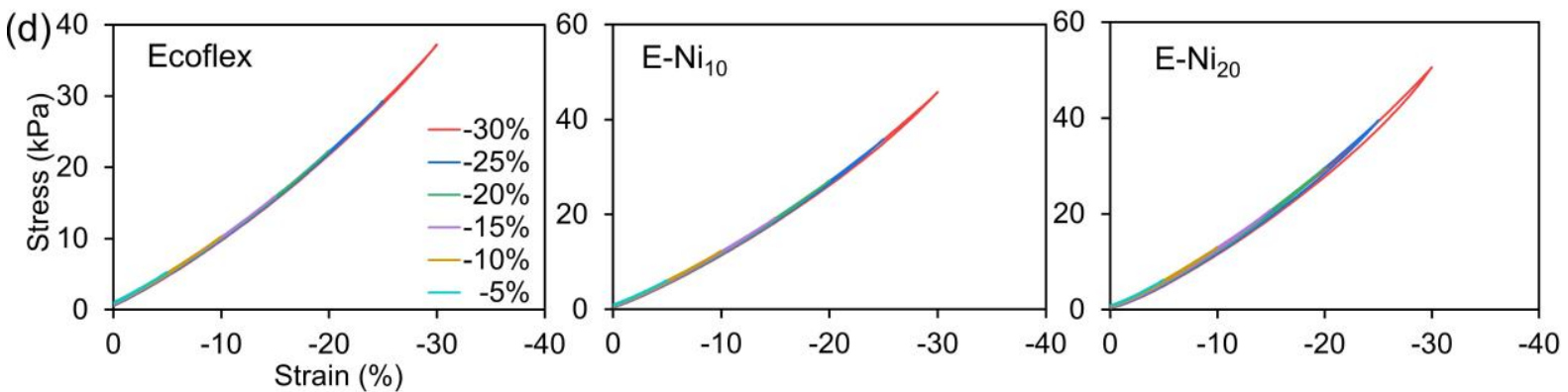

Figure S3. Cyclic loading tests for composites with different Ni particle ratios. a) Stress-strain curves for composite samples (Ecoflex, $\mathrm{E}-\mathrm{Ni}_{10}$, and $\mathrm{E}^{-\mathrm{Ni}_{20}}$, respectively; sample dimension: $10 \times 10 \times 10 \mathrm{~mm}$ ) under a compressive strain of $-20 \%$ in 6 cycles. No obvious elastic hysteresis is observed. b) Stress-strain curve for the E-Ni 30 sample under a compressive strain of $-20 \%$ in 6 cycles. Elastic hysteresis can be observed in this case. The inset enlarges the curve for the strain from $-13 \%$ to $-17 \%$. It is worth noting that the curve overlaps from second cycle, and the stress for the first cycle is larger than other cycles at the same strain. c) Stress-strain curves of the $\mathrm{E}-\mathrm{Ni}_{30}$ sample under increasing cyclic loadings (from $-5 \%$ to $-30 \%$ ). The enlarged inset shows the details for the strain from $-17 \%$ to $-26 \%$. This indicates that the $\mathrm{E}_{-\mathrm{Ni}} \mathrm{N}_{30}$ exhibits the Mullins effect. d) Stress-strain curves of Ecoflex, E-Ni ${ }_{10}$, and E-Ni ${ }_{20}$ samples under increasing cyclic loadings (from $-5 \%$ to $-30 \%$ ). The stress-strain curves for composites with particle contents below $20 \mathrm{wt} \%$ are independent of the maximum loadings in the history. 

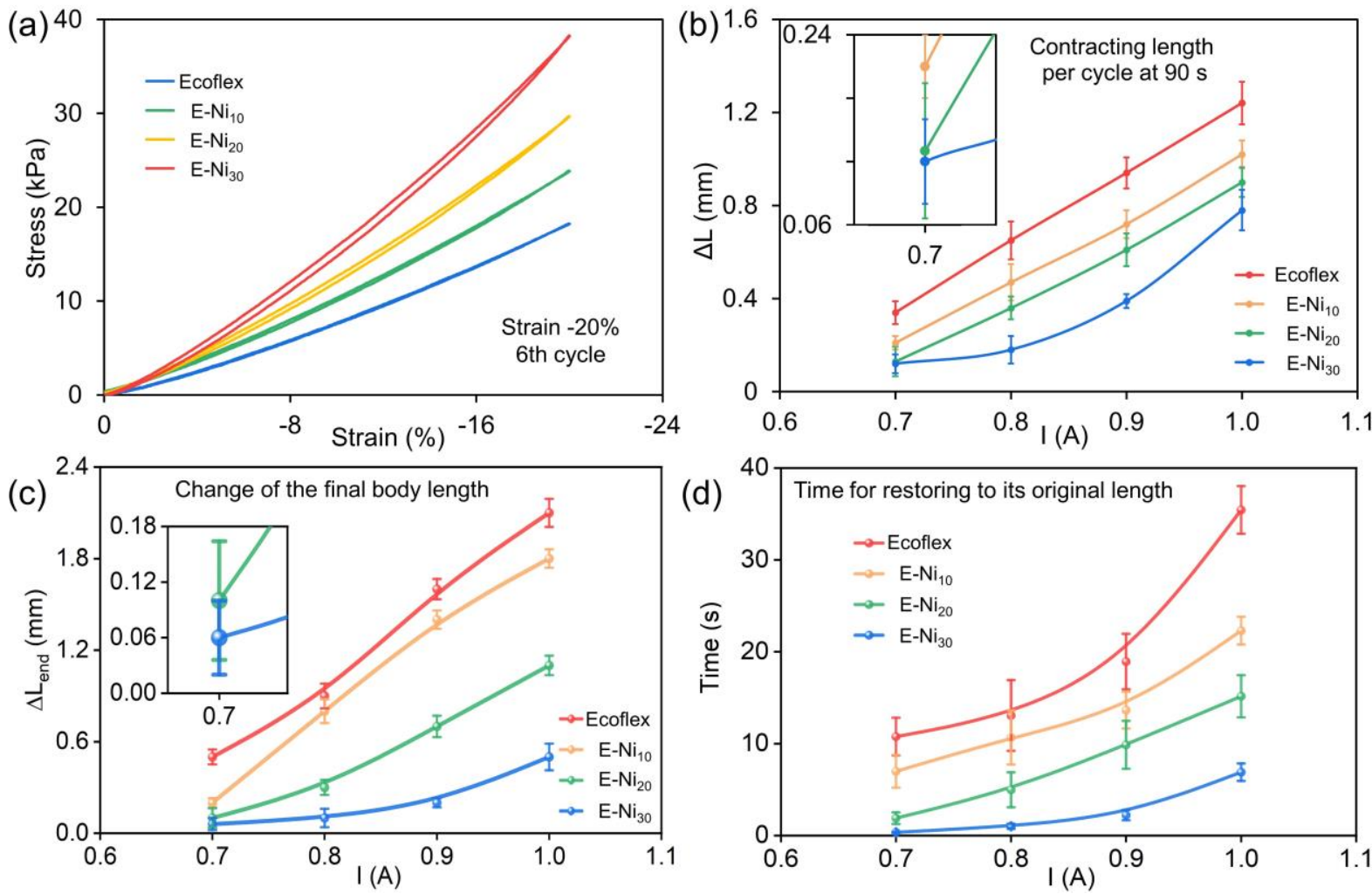

Figure S4. Characterization of the performance of the crawling robot fabricated by composites with different contents of Ni particles. a) Stress-strain curves of composites with different $\mathrm{Ni}$ contents at the 6th cycle. A higher Ni content leads to a higher stress at the same strain. $b$ ) Average contracting length per cycle $(\Delta L)$ of the robot body (length $\times$ width $\times$ height: $30 \times 6$ $\times 5 \mathrm{~mm}) 90 \mathrm{~s}$ after operating the robot with different levels of currents passing through the encapsulated SMA spring. The enlarged plot shows $\Delta L$ at 0.7 A. Composites with high $\mathrm{Ni}$ contents are more rigid, thereby resulting in smaller $\Delta L$. As we aim to increase the speed of robot, a large $\Delta L$ is desirable. c) Change of the final body length (for cases of Ecoflex, E-Ni $i_{10}$, $\mathrm{E}-\mathrm{Ni}_{20}, \mathrm{E}-\mathrm{Ni}_{30}$ ) after operating the robot for $3 \mathrm{~min}$ (contracting time of $1 \mathrm{~s}$ and restoring time of $2 \mathrm{~s}$ for each cycle) with different levels of currents. The enlarged inset shows the change of the length at the current of $0.7 \mathrm{~A}$. The robot body becomes shorter after experiments for all cases due to the accumulation of heat, which prolongs the restoring time of the SMA spring. d) Characterization of the time required for the robot body (for cases of Ecoflex, E-Ni 10 , E$\mathrm{Ni}_{20}, \mathrm{E}-\mathrm{Ni}_{30}$ ) to restore to its original length after working for $3 \mathrm{~min}$ at different levels of currents. The SMA spring requires a certain amount of time to cool down to release its tension.

In summary, $\mathrm{E}-\mathrm{Ni}_{20}$ combines desirable properties of good thermal conductivity, relatively large Young's modulus, and large contracting length, which was finally chosen to be used for making the body of the scrawling robot. 


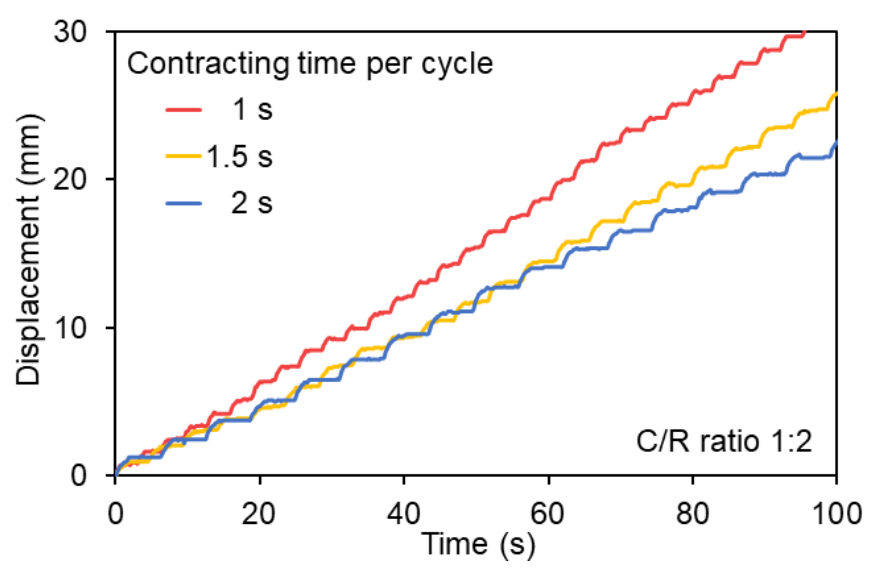

Figure S5. Displacement $v s$ time plots for the robot operated at the same $\mathrm{C} / \mathrm{R}$ ratio (1:2) but different contracting time. All cases can give a stable movement for the robot. A contracting time of $1 \mathrm{~s}$ leads to the fastest speed. 

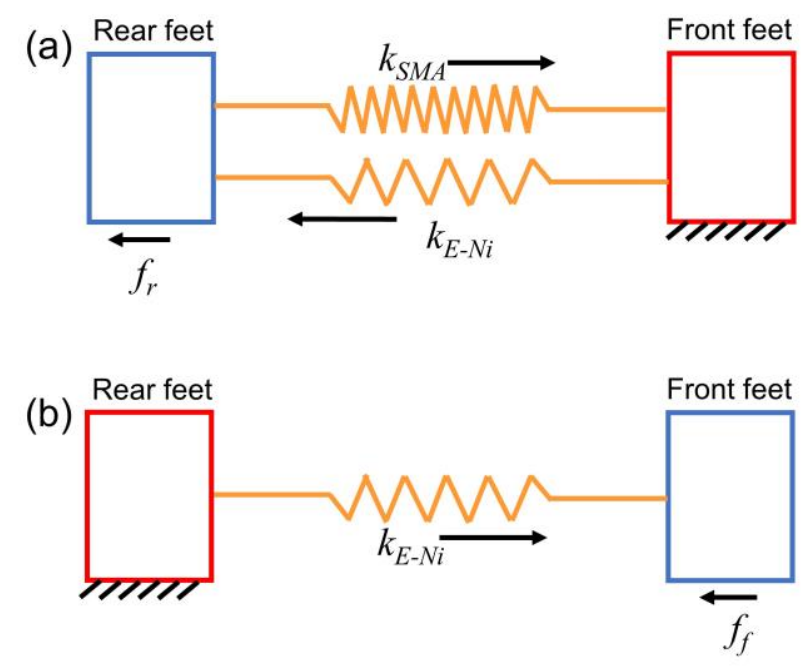

Figure S6. Theoretical model for the crawling soft robot during motion. a) Contracting force model for the rear LMSF. b) Restoring force model for the front LMSF.

Theoretically, kinetic friction of the front and rear LMSF can be calculated as:

$$
\begin{gathered}
f_{f}=\mu_{f} \frac{m g}{2} \\
f_{r}=\mu_{r} \frac{m g}{2}
\end{gathered}
$$

where $f_{r / f}$ and $\mu_{r / f}$ are the friction force and coefficient of friction for the rear and front LMSF, respectively. To ensure the front LMSF with higher friction keeps still on the surface while stroking for moving forward, the acceleration should meet the following constraint:

$$
a \leq \frac{f_{f}}{m}
$$

We can estimate the maximum acceleration that the front LMSF do not slip is $\sim 0.59 \mathrm{~m} \mathrm{~s}^{-2}$. From our experiments, we measured the initial acceleration of the rear LMSF to be $a_{r}=6.6$ $\mathrm{mm} \mathrm{s}^{-2}$, which is much smaller than the acceleration required for moving the front LMSF. 
Noticeably, there are two stages of the motion in one cycle, i.e., contracting and restoring. In the contracting stage, the rear LMSF moves forward, and its motion can be described as:

$$
\begin{gathered}
k_{S M A}\left(h-x_{C}\right)-k_{E-N i} x_{C}-f_{r}=\frac{1}{2} m \ddot{x_{C}} \\
x_{C}=\iint \ddot{x_{C}} d t d t \\
x_{C}(0)=\dot{x_{C}}(0)=0
\end{gathered}
$$

where $k_{S M A}$ and $k_{E-N i}$ are the stiffness of the SMA spring and the composite, respectively; $h$ denotes the length discrimination of the SMA between the initial state and the total contracting state; $f_{r}$ denotes the friction of the rear feet, which is negligible in this case; and $x_{C}$ is the displacement of the rear feet for contracting. $k_{S M A}$ varies when the SMA is heated as it transits from martensite to austenite. ${ }^{1}$ When the phase of the SMA is fully austenite, it can be regarded as purely elastic. In our experiments, the current passing through the SMA can sharply increase the temperature to achieve the full phase transition. Therefore, we can regard the $k_{S M A}$ as a constant.

Substituting the above conditions into Equation S1, the displacement of the rear feet versus time can be calculated as:

$$
x_{C}(t)=\frac{k_{S M A} h}{k_{S M A}+k_{E-N i}}\left(1-\cos \sqrt{\frac{2\left(k_{S M A}+k_{E-N i}\right)}{m}} t\right)
$$

where $t$ denotes the time during contraction. For the restoring stage, we postulate that the SMA fully transits into martensite phase due to the rapid drop of temperature. In this case, 
SMA can be regarded as plastic and $k_{S M A}$ is negligible (see Figure S6b). The motion of the front LMSF can be expressed as:

$$
\begin{gathered}
k_{E-N i}\left(x_{C-\text { total }}-x_{R}\right)-f_{f}=\frac{1}{2} m \ddot{x_{R}} \\
x_{R}=\iint \ddot{x_{R}} d t d t \\
x_{R}(0)=\dot{x_{R}}(0)=0 \\
x_{C-\text { total }}=\frac{k_{S M A} h}{k_{S M A}+k_{E-N i}}\left(1-\cos \sqrt{\frac{2\left(k_{S M A}+k_{E-N i}\right)}{m}} t_{C}\right)
\end{gathered}
$$

where $t_{C}$ is the total contracting time, $x_{C \text {-total }}$ is the total displacement of the rear feet during the contracting stage, and $x_{R}$ is the displacement of the front feet. Similarly, $f_{f}$ is negligible due to the slip layer when the LMSF is reduced. Substituting the above conditions into Equation S8, the displacement of the rear feet can be calculated as:

$$
x_{R}(t)=\frac{k_{S M A} h}{k_{S M A}+k_{E-N i}}\left(1-\cos \sqrt{\frac{2\left(k_{S M A}+k_{E-N i}\right)}{m}} t_{C}\right)\left(1-\cos \sqrt{\frac{2 k_{E-N i}}{m}} t\right)
$$


(a)

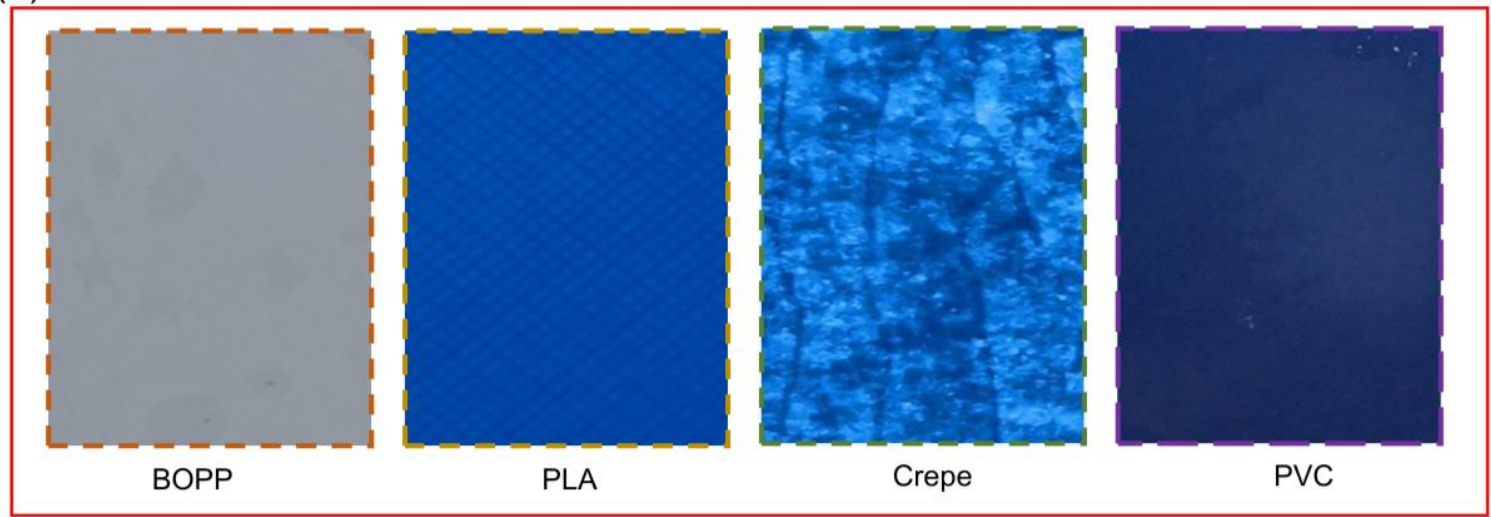

(b)

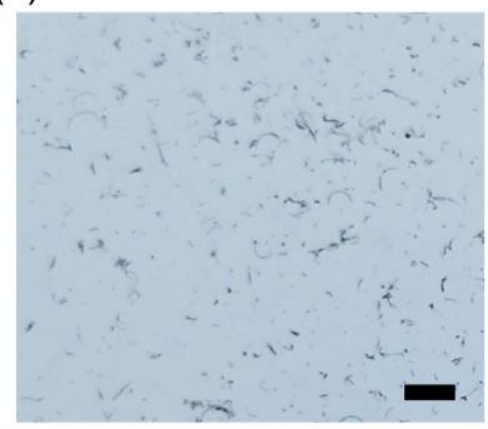

(c)

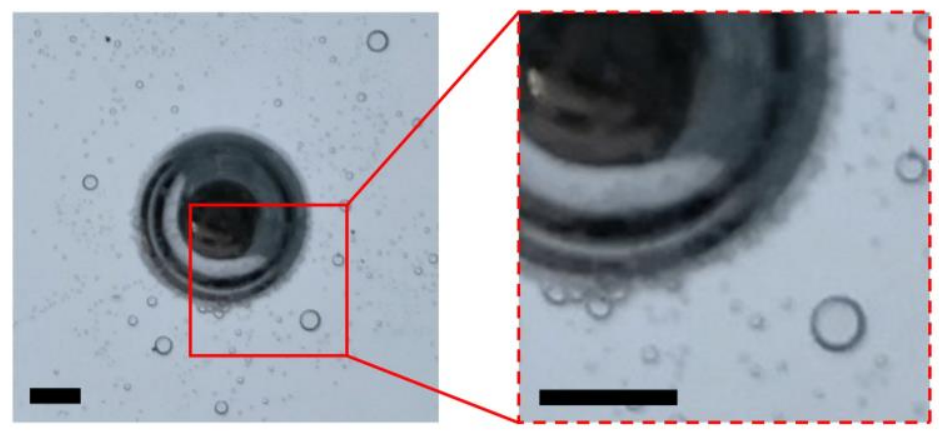

Figure S7. Operation of the LMSF on different substrates and in different solutions. a) Images of the surface of different substrates. b) Optical image of the black floccule when operating the LMSF in a $\mathrm{NaCl}$ solution. c) Generation of gas bubbles around a LM droplet in a $\mathrm{HCl}$ solution. Scale bars are $2 \mathrm{~mm}$. 

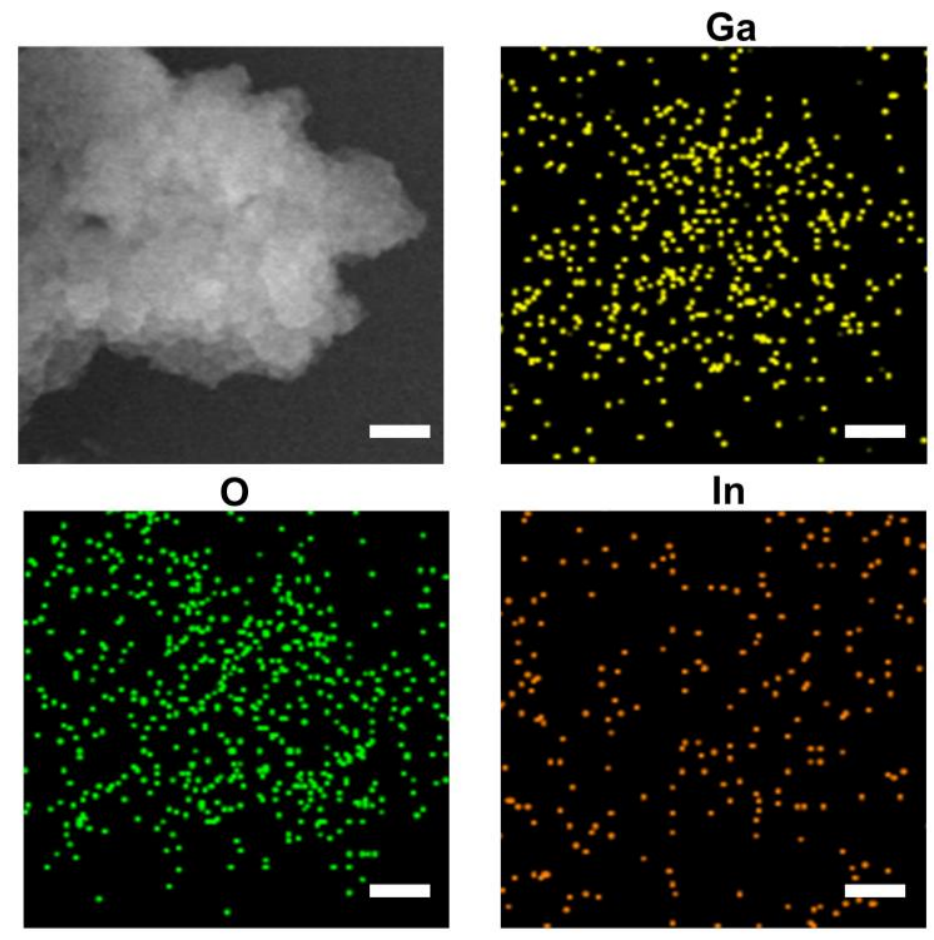

In

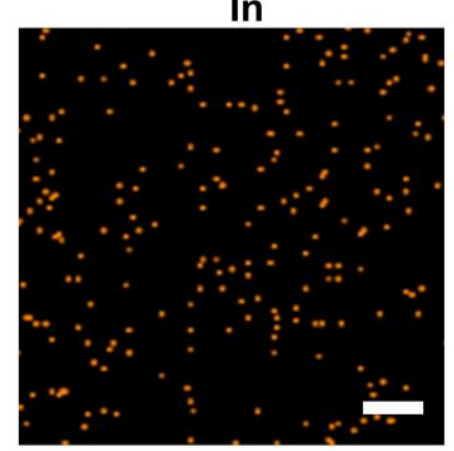

Figure S8. EDS mappings of the floccule generated around the LMSF operated in seawater. Scale bars are $500 \mathrm{~nm}$. 

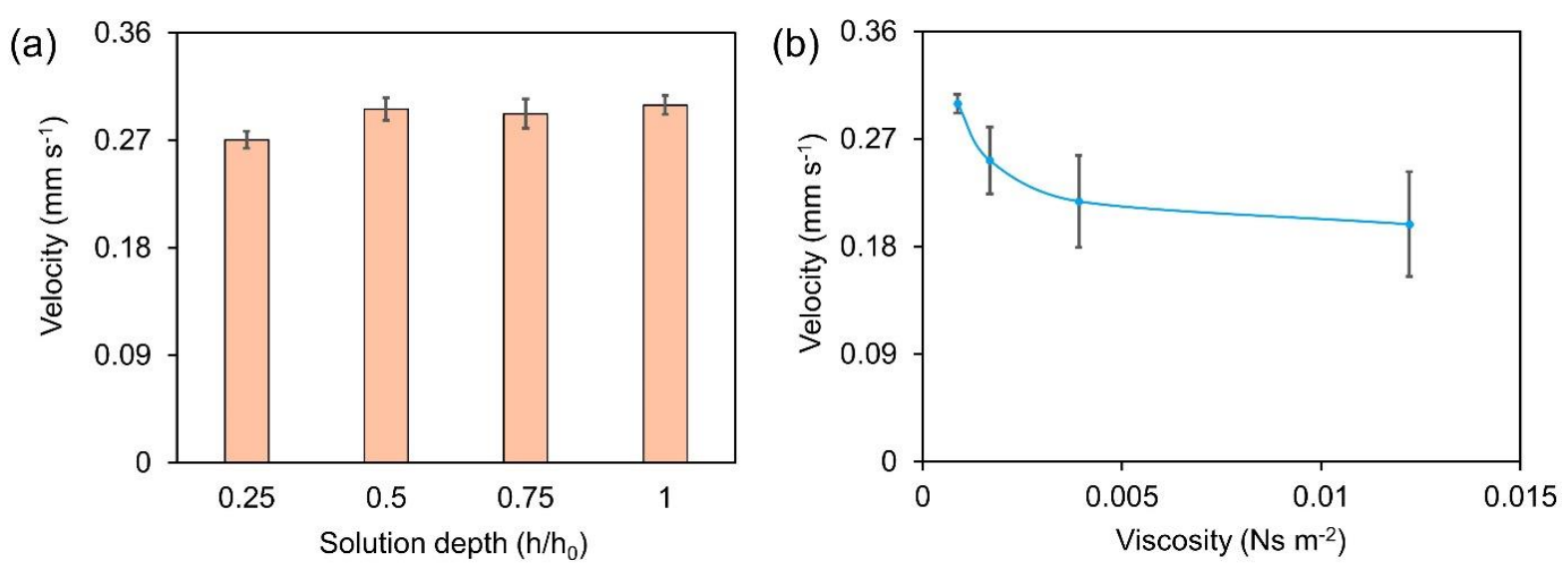

Figure S9. Performance of the LMSF in the solutions with different depths and viscosities. a). Comparison of actuation performance in $\mathrm{NaOH}$ solutions $\left(0.5 \mathrm{~mol} \mathrm{~L}^{-1}\right)$ with different depths, where $h_{0}$ is the height of the LMSF and $h$ is the depth of solution. b) Characterization of actuation performance in solutions of different viscosities.

Figure S9a shows that no obvious difference in speed was observed when actuating in solutions of different speeds. To investigate the effect of viscosity, we mixed glycerol with $\mathrm{NaOH}$ solutions at volume ratios of $20 / 40 / 60 \%$ to prepare solutions with different viscosities. ${ }^{1}$ The concentration of $\mathrm{NaOH}$ was kept constant at $0.5 \mathrm{~mol} \mathrm{~L}^{-1}$. Figure $\mathrm{S} 9 \mathrm{~b}$ shows that the actuating velocity decreased by $\sim 34 \%$ when the viscosity of the solution was increased by 14 times. This result is expected since a larger drag is induced in a solution with a higher viscosity. 


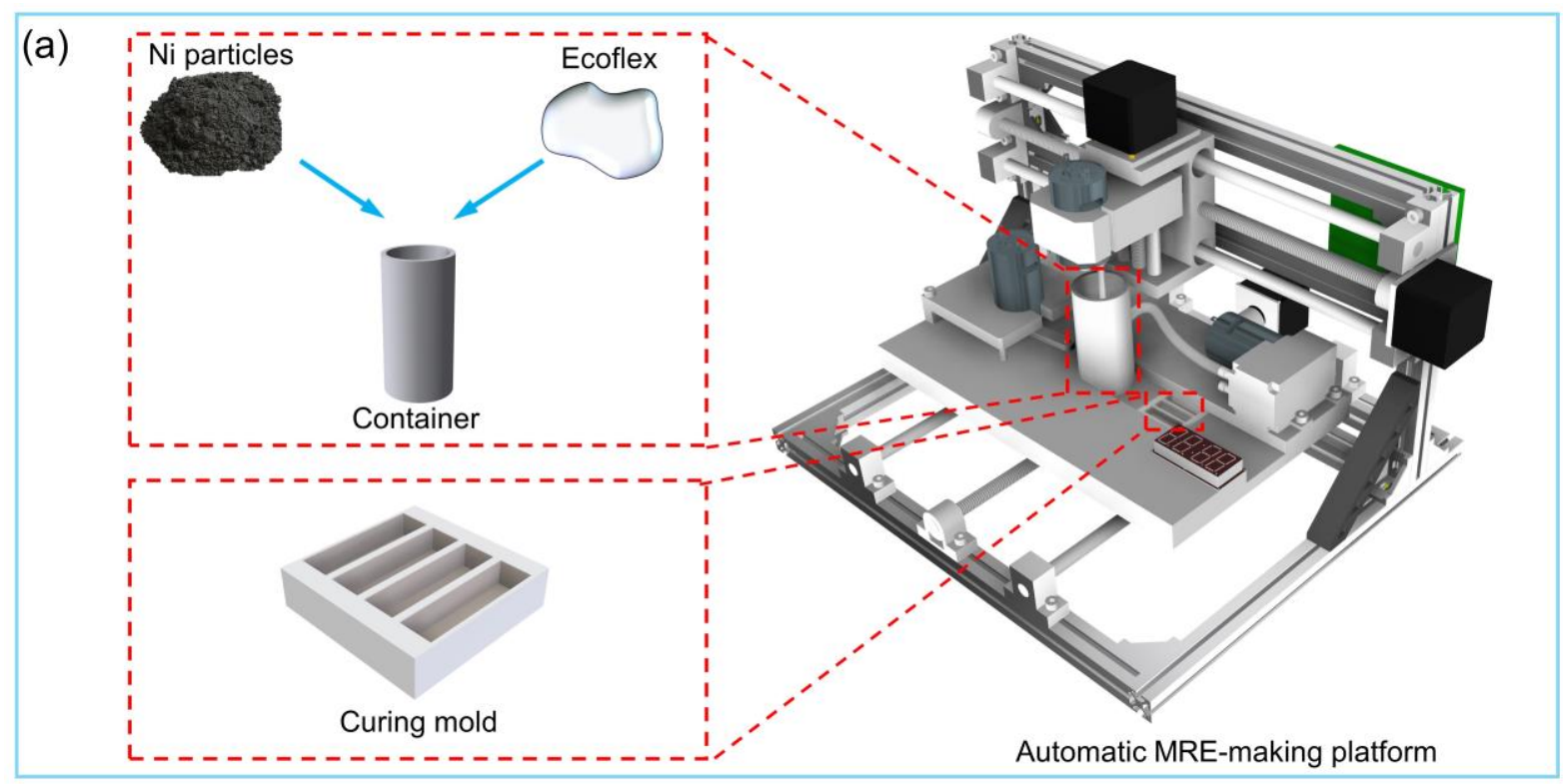

(b)

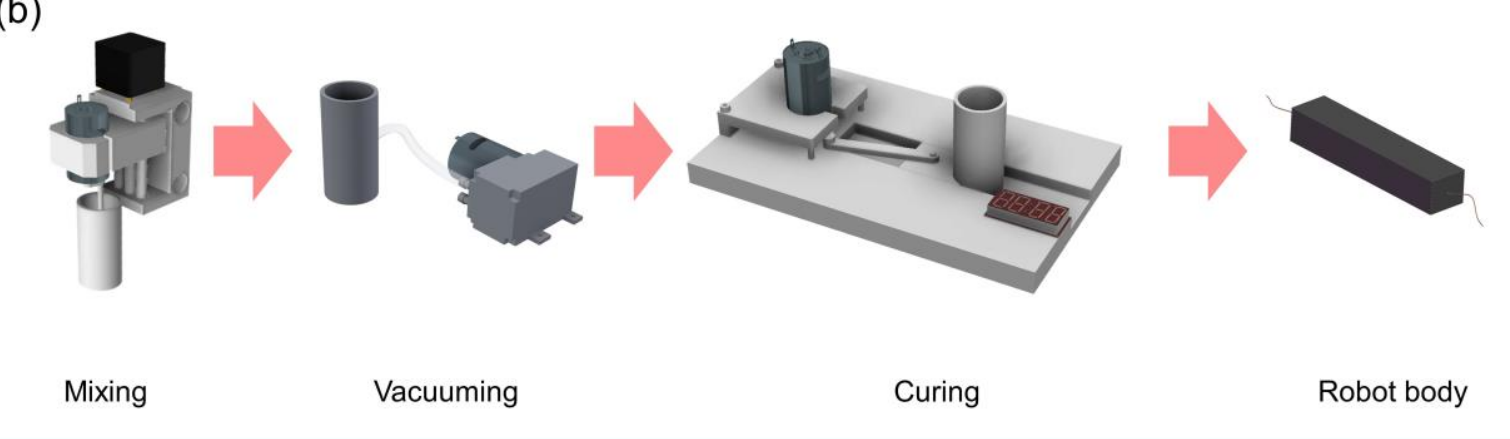

Figure S10. Fabrication process of the robot body $\left(E-\mathrm{Ni}_{\mathrm{x}}, \mathrm{x}=10 / 20 / 30\right)$. a) Schematic of the automatic magnetorheological elastomer fabrication platform. The upper left enlarged image displays the container in which the raw materials are poured in. The lower left inset shows the curing mold. b) Fabricating process for the body of the crawling robot.

We designed an automatic magnetorheological elastomer fabrication platform to make the robot body for keeping batch-to-batch consistency (Figure S10a). Such a platform consists a 3D moving platform, a slider-crank mechanism, a materials-mixing module, a vacuum pump, a temperature control system, and a control module. The fabricating process includes mixing, vacuuming, and curing, as shown in Figure S10b. We firstly weigh raw materials and poured them in the container. Next, the platform can automatically fabricate the body of the crawling 
robot following the programed order. After mixing raw materials thoroughly for $3 \mathrm{~min}$, the vacuum pump starts to remove the air bubbles in the composite. 5 min later, the container is pushed towards the curing mold. The temperature control system heats the composite and maintains it at $60^{\circ} \mathrm{C}$ for $2 \mathrm{~h}$ while the LED displays the real-time temperature. Finally, we can peel the cured composite from the mold.

\section{Supporting Information Reference}

(1) Brinson, L. C. One-Dimensional Constitutive Behavior of Shape Memory Alloys: Thermomechanical Derivation with Non-Constant Material Functions and Redefined Martensite Internal Variable. J. Intell. Mater. Syst. Struct. 1993, 4 (2), 229-242, DOI: $10.1177 / 1045389 X 9300400213$. 